\title{
Eigenfunction Expansions Associated with the Schrödinger Operators with Long-Range Potentials
}

By

\author{
Masaharu ARAI*
}

\section{§1. Introduction}

The present paper is devoted to constructing eigenfunction expansions associated with the Schrödinger operator

$$
L=-\Delta+V(x), x \in R^{n}(n \geqq 2),
$$

where $\Delta$ denotes the Laplacian: $\Delta=\sum_{j=1}^{n}\left(\partial^{2} / \partial x_{j}^{2}\right)$, and $V(x)$ is a real valued continuous function. We interpret for the time being, the eigenfunction expansion associated with a selfadjoint realization $H$ of $L$ as follows: select suitable function $\phi=\phi(x ; \lambda, \alpha)$, where $\alpha$ runs over a set $\Omega_{\lambda}$ of indeces, out of the solutions of the equation

$$
L \phi=\lambda \phi
$$

in the distribution sense and construct a measure $d \rho$ on $\Omega=\{(\lambda, \alpha)\}$ such that the operator $\mathbb{F}$ defined by

$$
(\mathscr{I} f)(\lambda, \alpha)=\int f(x) \overline{\phi(x ; \lambda, \alpha)} d x
$$

has the following properties:

(1) $\mathscr{F}$ is unitary from $L_{2}\left(R^{n} ; d x\right)$ onto $L_{2}(\Omega ; d \rho)$.

(2) $(\mathscr{H}(H f))(\lambda, \alpha)=\lambda(\mathscr{H} f)(\lambda, \alpha)$.

General frameworks for formulating eigenfunction expansions have been proposed by several authors, for example by Gel'fand et al. [2], [3], which can be applied to the Schrödinger operator $L$ with smooth potential $V$ in a certain large class. They have not specified the eigenfunctions needed for expansions in

Communicated by S. Matsuura, March 26, 1977. Revised May 12, 1978.

* Faculty of Economics, Ritsumeikan University. 
terms of a conditions (depending on $\alpha$ ) under which each of them is the unique solution of (1.2). In addition, their measure $d \rho$ is devided from the spectral resolution associated with $H$ so that it is less explicit.

Another approach is of a parturbation theoretic charactor, in which one can adopt as the measure $d \rho$ the same one as that of $L$ with $V=0$. Assume that

$$
V(x)=O\left(|x|^{-\delta}\right) \quad \text { as }|x| \rightarrow \infty .
$$

The eigenfunction expansions in this sense are obtained by Ikebe [5] in the case of $\delta>2$ and $n=3$, by Thoe [17] and Kuroda [13] in the case of $\delta>(n+1) / 2$. (Of cause, all of them allow local singularities in their respective sense. But we will not enter this point into details.) Put $\phi_{1}(x, \zeta)=(2 \pi)^{-n / 2} e^{i \zeta \cdot x}, \zeta \in R^{n}$. Then the limiting absorption principle, cf [9; Theorem 1.4], implies that the limits

$$
R\left(|\zeta|^{2} \pm i 0\right)=\lim _{\mu \downarrow 0}\left[H-\left(|\zeta|^{2} \pm i \mu\right)\right]^{-1}
$$

exist in a certain sence and we can define

$$
\phi^{ \pm}(\cdot ; \zeta)=\phi_{1}(\cdot ; \zeta)-R\left(|\zeta|^{2} \mp i 0\right) V \phi_{1}(\cdot ; \zeta)
$$

if $\delta>(n+1) / 2$. A somewhat long calculation shows that these two families $\left\{\phi^{ \pm}(\cdot ; \zeta)\right\}$ give the eigenfunction expanisons with $\Omega=R^{n}, \lambda=|\zeta|^{2}$ and $d \rho=d \zeta$ the ordinary Lebesque measure. Moreover the limiting absorption principle cited above shows that these eigenfunctions are the unique solutions of (1.2) under the condition

$$
\begin{aligned}
& \left\{\begin{array}{l}
(1+|x|)^{-1-\varepsilon}\left\{\phi^{ \pm}(x ; \zeta)-\phi_{1}(x: \zeta)\right\} \in L_{2}\left(R_{x}^{n}\right) \\
(1+|x|)^{-1+\varepsilon}\{\partial / \partial|x|+(n-1) / 2|x| \pm i|\zeta|)\left(\phi^{ \pm}(x: \zeta)-\phi_{1}(x ; \zeta)\right)
\end{array}\right. \\
& \in L_{2}(x ;|x| \geqq 1)
\end{aligned}
$$

for sufficiently small $\varepsilon>0$.

In the case of $\delta>1$ in (1.3), Agmon [1] gives the eigenfunctions, which seems to satisfy merely a weaker version of condition (1.5) (cf. (5.17) of [1]). Recently, Pinchuk [14] have obtained the eigenfunctions satisfying a modified virsion of the condition (1.5) under the assumptions that $n=3$ and $V$ can be decomposed as $V=V_{1}+V_{2}$ such that

$$
\left\{\begin{array}{l}
\left|V_{1}(x)\right| \leqq C(1+|x|)^{-\delta} \\
\left|\operatorname{grad} V_{1}(x)\right| \leq C(1+|x|)^{-1-\delta} \\
\left|\Lambda V_{1}(x)\right| \leq C(1+|x|)^{-\delta} \\
\left|V_{2}(x)\right| \leqq C(1+|x|)^{-\delta^{\prime}}
\end{array}\right.
$$


for $\delta>1$ and $\delta^{\prime}>2$, where $\Lambda$ is the Laplace-Beltrami operator on the unit sphere $S^{n-1}$ in $R^{n}$.

On the other hand, Kato and Kuroda [12] have pointed out the eigenfunction expansion theorem for $\delta>1$ in (1.3), where eigenfunctions are given by (1.4) with $\phi_{1}(x ; \zeta)$ replaced by the spherical waves, which will be defined by (3.3.1).

The aim of this paper is to obtain the eigenfunction expansion whose each eigenfunction satisfies a modified virsion of (1.5), i.e. (4.1.3), with $\phi_{1}(x ; \zeta)$ replaced by the spherical waves under the condition that $\delta>1 / 2$ and $\delta^{\prime}>1$ in (1.6) without the condition on $\Lambda V_{1}$. Our plan of this paper is as follows. In Section 2, we shall sketch an abstract method, most part of which we owe to Isozaki [10]. He extended Ikebe's work in [6] to a fashion to be applicable to the longrange potentials and used it to construct the wave operators. In Section 3, we shall check up that the abstract theory given in Section 2 is applicable to the Schrödinger operators. In Section 4, we shall introduce a modified virsion of condition (1.3) and show that the equation (1.2) has the unique solution satisfying this condition and totality of such unique solutions gives eigenfunction expansion theorem. In Section 5, we shall prove two lemmas which will be used in Secticn 4.

As to the eigenfunction expansion associated with the Schrödinger operator with long-range potentials we should mention to the work by Ikebe [7], [8] and Saitō [15], [16].

In conclusion, the writer wishes to express his sincere gratitude to Professor $\mathrm{T}$. Ikebe for his kind advice. He also thanks to $\mathrm{Mr}, \mathrm{H}$. Isozaki for his kind advice.

\section{§2. Abstract Theory}

2.1. Let $\mathcal{H}$ be a separable Hilbert space. Let $H_{j}(j=1,2)$ be selfadjoint operators in $\mathcal{H}$, whose spectral resolutions are denoted by $E_{j}(\lambda)$ and whose resolvent operators by $R_{j}(z)=\left(H_{j}-z\right)^{-1}$.

Let $\widetilde{\mathscr{H}}_{+}$and $\mathscr{H}_{+}$be Hilbert spaces such that

$$
\widetilde{\mathscr{H}}_{+} \subset \mathscr{H}_{+} \subset \mathcal{H}
$$

and all inclusions are dense and continuous. Their dual spaces are denoted by $\widetilde{\mathcal{H}}_{-}$and $\mathscr{H}_{-}$, respectively. We identify $\mathscr{H}$ with its dual space so that we have

$$
\tilde{\mathscr{H}}_{+} \subset \mathscr{H}_{+} \subset \mathscr{H} \subset \mathscr{H}_{-} \subset \tilde{\mathscr{H}}_{-} .
$$

We use $($, ) to denote not only the inner product of $\mathscr{H}$ but also the pairing of 
$\widetilde{\mathscr{H}}_{+}$and $\widetilde{\mathscr{H}}_{-}$, and of $\mathscr{H}_{+}$and $\mathscr{H}_{-}$, which will not confuse our arguments. We denote by $\mathscr{B}\left(A_{1}, A_{2}\right)$ the totality of bounded linear operators from a Hilbert space $A_{1}$ to a Hilbert space $A_{2}$.

2.2. We assume that the limiting absorption principle is guaranteed in the following sense.

Assumption 2.2.1. $R_{j}(\lambda \pm i \mu) \in \mathscr{B}\left(\mathscr{H}_{+}, \mathscr{H}_{-}\right)$for any $\lambda>0$ and $\mu>0$, and the strong limit

$$
R_{j}(\lambda \pm i 0)=s-\lim _{\mu \downarrow 0} R_{j}(\lambda \pm i \mu)
$$

exists. Moreover, $R_{j}(\lambda \pm i \mu) f$ is an $\mathscr{H}_{-}$-valued strongly continuous functions of $(\lambda, \mu) \in(0, \infty) \times[0, \infty)$ for any $f \in \mathscr{H}_{+}(j=1,2)$.

With the aid of this assumption we define

$$
E_{j}^{\prime}(\lambda)=\frac{1}{2 \pi i}\left[R_{j}(\lambda+i 0)-R_{j}(\lambda-i 0)\right] \quad(j=1,2) .
$$

which belongs to $\mathscr{B}\left(\mathscr{H}_{+}, \mathscr{H}_{-}\right)$.

Let $H$ be a selfadjoint operator in $\mathscr{H}$ and $E(\lambda)$ be its spectral resolution. The set $\mathscr{H}_{a c}(H)$ defined by

$\mathscr{H}_{a c}(H)=\{f \in \mathscr{H} ;(E(\lambda) f, f)$ is absolutely continuous function of $\lambda$ with respect to the Lebesque measure\},

which is a closed subspace of $\mathscr{H}$ (see e.g. [11; Chap. X]). $H$ is called absolutely continuous if and only if $\mathscr{H}_{a c}(H)=\mathscr{H}$.

Lemma 2.2.2. Let Assumption 2.2 .1 be satisfied. Then $E_{j}((0, \infty)) H_{j}$ is absolutely continuous.

Proof. Let $f \in \mathscr{H}_{+}$and $\Delta=\left(\lambda_{1}, \lambda_{2}\right)$, where $0<\lambda_{1}<\lambda_{2}<\infty$. As is well known it holds that

$$
\left(E_{j}(\Delta) f, f\right)=\lim _{\varepsilon_{\ddagger} 0} \lim _{\psi_{\ddagger} 0} \frac{1}{2 \pi i} \int_{\lambda_{1}+\varepsilon}^{\lambda_{2}-\varepsilon}\left(\left\{R_{j}(\lambda+i \mu)-R_{j}(\lambda-i \mu)\right\} f, f\right) d \lambda .
$$

Since the integrand is continuous function of $(\lambda, \mu) \in \bar{\Delta} \times[0,1]$, we can use dominated convergence theorem to obtain

$$
\left(E_{j}(\Delta) f, f\right)=\int_{\Delta}\left(E_{j}^{\prime}(\lambda) f, f\right) d \lambda,
$$

which shows that $\mathscr{H}_{+} \subset \mathscr{H}_{a c}(H)$. Since $\mathscr{H}_{+}$is dense in $\mathscr{H}_{\text {and }} \mathscr{H}_{a c}(H)$ is closed in $\mathscr{H}$, we have the present lemma. 
Our next assumption is:

Assumption 2.2.3. There exist unitary operators $U_{k j}^{ \pm}(\lambda)(\lambda>0)$ on $\mathscr{H}$ having the following properties.

(1) $U_{k j}^{ \pm}(\lambda)^{*}=U_{j k}^{ \pm}(\lambda)$.

Here and in the sequel $j, k=1,2$ and $j \neq k$.

(2) $U_{k j}^{+}(\lambda) R_{j}(\lambda \pm i \mu) f \in \mathscr{D}\left(H_{k}\right)$ for any $f \in \mathcal{H}$ and $\lambda, \mu>0$.

(3) The operator $G_{j k}(\lambda \pm i \mu)(\lambda, \mu>0)$ defined by

$$
G_{j k}(\lambda \pm i \mu)=\left[H_{j}-(\lambda \pm i \mu)\right] U_{j k}^{ \pm}(\lambda) R_{k}(\lambda \pm i \mu)
$$

belongs to $\mathcal{B}\left(\widetilde{\mathscr{H}}_{+}, \mathscr{H}_{+}\right)$and the strong limit

$$
G_{j k}(\lambda \pm i 0)=s-\lim _{\mu_{\ddagger} 0} G_{j k}(\lambda \pm i \mu)
$$

exists in $\mathcal{B}\left(\widetilde{\mathscr{H}}_{+}, \mathscr{H}_{+}\right)$. Moreover, $G_{j k}(\lambda \pm i 0) f$ is a strongly continuous function of $\lambda>0$ for any $f \in \widetilde{\mathscr{H}}_{+}$.

Let $e$ be a bounded Borel set contained in $(0, \infty)$ and having a positive distance from 0 . We define

$$
W_{j k}^{ \pm}(e) f=\int_{e} E_{j}^{\prime}(\lambda) G_{j k}(\lambda \pm i 0) f d \lambda, f \in \widetilde{\mathcal{H}}_{+} .
$$

By virtue of Assumptions 2.2.1 and 2.2.3, this integral is well defined and $W_{j k}^{ \pm}(e) \in \mathcal{B}\left(\tilde{\mathscr{H}}_{+}, \mathscr{H}_{-}\right)$. Moreover, we have

Theorem 2.2.4. ([10]) Assume that Assumptions 2.2.1 and 2.2.3 are satisfied. Then,

(1) The operator $W_{j k}^{ \pm}(e)$ is uniquely extended to partial isometry on $\mathscr{H}$ with initial set $E_{k}(e) \mathscr{H}$ and final set $E_{j}(e) \mathcal{H}$. (We use the same notation for its extension.)

(2) $W_{j k}^{ \pm}(e)^{*}=W_{k j}^{ \pm}(e)$.

(3) $W_{j k}^{+}(e)$ intertwines $H_{j}$ and $H_{k}$. That is,

$$
E_{j}(\Delta) W_{j k}^{ \pm}(e)=W_{j k}^{ \pm}(e) E_{k}(\Delta)
$$

for any Borel set $\Delta \subset e$

For the proof of this theorem, see [10].

2.3. We assume that the eigenfunction expansion on $e$ associated with $H_{1}$ is guaranteed in the following sense. 
Assumption 2.3.1.

(1) There are a $\sigma$-finite measure space $(\Omega, \Sigma, d \rho)$, a partial isometry $\mathscr{F}_{1}$ from $\mathcal{H}$ onto $L_{2}(\Omega, d \rho)$ with initial set $E_{1}(e) \mathcal{H}$, and a measurable function $\omega: \Omega$ $\rightarrow e$ such that

$$
\left(\mathscr{F}_{1} E_{1}(\Delta) f\right)(\zeta)=\chi_{\Delta}(\omega(\zeta))\left(\mathscr{F}_{1} f\right)(\zeta) \quad \text { a.e. } \zeta \in \Omega
$$

for each $f \in \mathcal{H}$ and Borel set $\Delta \subset e$, where $\chi_{\Delta}$ is the characteristic function of set $\Delta$.

(2) There is a mapping $\phi_{1}: \Omega \rightarrow \mathscr{H}_{-}$such that

$$
\left(\mathscr{F}_{1} f\right)(\zeta)=\left(f, \phi_{1}(\zeta)\right)
$$

for each $f \in \mathscr{H}_{+}$.

Theorem 2.3.2. Assume that Assumptions 2.2.1, 2.2.3 and 2.3.1 are satisfied. We put

$$
\phi_{2}^{ \pm}(\zeta)=G_{12}^{\dagger}(\omega(\zeta) \pm i 0) \phi_{1}(\zeta)
$$

and

$$
\left(\mathscr{F} \frac{ \pm}{2} f\right)(\zeta)=\left(f, \phi_{2}^{ \pm}(\zeta)\right) \quad f \in \widetilde{\mathcal{H}}_{+},
$$

where $G_{12}^{\dagger}: \mathscr{H}_{-} \rightarrow \tilde{\mathcal{H}}_{-}$is the adjoint operator to $G_{12} \in \mathscr{B}\left(\tilde{\mathcal{H}}_{+}, \mathscr{H}_{+}\right)$. Then we have

$$
\mathscr{F}_{2}^{\frac{ \pm}{2}}=\mathscr{F}_{1} W_{12}^{ \pm}(e)
$$

on $\tilde{\mathcal{H}}_{+} . \quad$ Since the right hand side of (2.3.5) are defined on $\mathcal{H}$, we can uniquely extend the operator $\mathscr{F}_{\frac{1}{2}}^{+}$on $\mathscr{H}$ by this equation. This extension will be denoted by the same notation. Then we have

$$
\left(\mathscr{F}_{\frac{2}{2}}^{ \pm} E_{2}(\Delta) f\right)(\zeta)=\chi_{\Delta}(\omega(\zeta))\left(\mathscr{F}_{2}^{ \pm} f\right)(\zeta) \quad \text { a.e. } \zeta \in \Omega
$$

that is, Assumption 2.3.1 holds true with $\mathscr{F}_{1}, \phi_{1}, E_{1}$ and $\mathscr{H}_{+}$replaced by $\mathscr{F}_{2}^{ \pm}, \phi_{2}, E_{2}$ and $\widetilde{\mathcal{H}}_{+}$, respectively, and with the same $(\Omega, \Sigma, d \rho)$ and $\omega$.

Proof. Since $\phi_{1}$ is $\mathscr{H}_{-}$-valued and $G_{1_{2}}^{\dagger} \in \mathscr{B}\left(\mathcal{H}_{-}, \widetilde{\mathcal{H}}_{-}\right), \phi_{2}^{ \pm}$defined by (2.3.3) is $\widetilde{\mathscr{H}}_{-}$-valued so that (2.3.4) is well defined. Assume at first that the equation (2.3.5) has been proven. Then, the assumptions (2.3.5), (2.3.1) and (2.2.4) imply the assertion (2.3.6).

Let us prove (2.3.5) on $\tilde{\mathscr{H}}_{+}$. Let $f(\lambda)$ be

$$
f(\lambda)=\left\{\begin{array}{l}
f,(\lambda \in \Delta \subset e) \\
0,(\lambda \notin \Delta), \quad f \in \mathscr{H}_{+} .
\end{array}\right.
$$


Then we have

$$
\int_{e} E_{1}^{\prime}(\lambda) f(\lambda) d \lambda=\int_{\Delta} E_{1}^{\prime}(\lambda) f d \lambda=E_{1}(\Delta) f
$$

and

$$
\mathscr{F}_{1} \int_{e} E_{1}^{\prime}(\lambda) f(\lambda) d \lambda=\chi_{\Delta}(\omega(\zeta))\left(\mathscr{F}_{1} f\right)(\zeta)=\left(f(\omega(\zeta)), \phi_{1}(\zeta)\right) .
$$

Next, let $f(\lambda)$ be an $\mathscr{H}_{+}$-valued bounded continuous function defined on $e$, and approximate it by step functions $f_{m}(\lambda)$ such that $f_{m}(\lambda) \rightarrow f(\lambda)$ in $\mathscr{H}_{+}$for each $\lambda$. Then, using (2.3.7) it is easy to see that

$$
\begin{aligned}
& \left\|\int_{e} E_{1}^{\prime}(\lambda) f_{m}(\lambda) d \lambda-\int_{e} E_{1}^{\prime}(\lambda) f_{n}(\lambda) d \lambda\right\|^{2} \\
= & \int_{e}\left(E_{1}^{\prime}(\lambda)\left(f_{m}(\lambda)-f_{n}(\lambda)\right), f_{m}(\lambda)-f_{n}(\lambda)\right) d \lambda,
\end{aligned}
$$

which tends to zero as $m, n \rightarrow \infty$. Thus the sequence $\left\{\int_{e} E_{1}^{\prime}(\lambda) f_{m}(\lambda) d \lambda\right\}$ has a strong limit in $\mathcal{H}$ as $m \rightarrow \infty$. On the other hand it converges to $\int_{e} E_{1}^{\prime}(\lambda) f(\lambda) d \lambda$ in $\mathscr{H}_{-}$. Thus we have

$$
\int_{e} E_{1}^{\prime}(\lambda) f_{m}(\lambda) d \lambda \rightarrow \int_{e} E_{1}^{\prime}(\lambda) f(\lambda) d \lambda \text { in } \mathscr{H},
$$

and so we have

$$
\mathscr{F}_{1} \int_{e} E_{1}^{\prime}(\lambda) f_{m}(\lambda) d \lambda \rightarrow \mathscr{F}_{1} \int_{e} E_{1}^{\prime}(\lambda) f(\lambda) d \lambda \text { in } \mathscr{H} .
$$

Therefore we have

$$
\mathscr{F}_{1} \int_{e} E_{1}^{\prime}(\lambda) f(\lambda) d \lambda=\left(f(\omega(\zeta)), \phi_{1}(\zeta)\right) .
$$

Put $f(\lambda)=G_{12}(\lambda \pm i 0) f, f \in \widetilde{\mathscr{H}}_{+}$into the above equation. Then we have

$$
\begin{aligned}
\mathscr{F}_{1} W_{12}^{ \pm}(e) f & =\mathscr{F}_{1} \int_{e} E_{1}^{\prime}(\lambda) G_{12}(\lambda \pm i 0) f d \lambda \\
& =\left(G_{12}(\omega(\zeta) \pm i 0) f, \phi_{1}(\zeta)\right) \\
& =\left(f, \phi_{2}^{ \pm}(\zeta)\right)=\mathscr{F}_{2}^{ \pm} f,
\end{aligned}
$$

which is what we want to prove.

Q.E.D.

Lemma 2.3.3. Assume that the assumptions in Theorem 2.3 .2 are satisfied. Moreover, assume that there exists a set $\mathscr{D} \subset \tilde{\mathscr{H}}_{+} \cap \mathscr{D}\left(H_{2}\right)$ such that $H_{2} \mathscr{D} \subset \tilde{\mathscr{H}}_{+}$. Then we have

$$
\left(H_{2} \varphi, \phi_{2}^{ \pm}(\zeta)\right)=\omega(\zeta)\left(\varphi, \phi_{2}^{ \pm}(\zeta)\right)
$$


for any $\varphi \in \mathscr{D}$.

Proof. The identity

$$
\left(H_{2} \varphi, \phi_{2}^{ \pm}(\zeta)\right)=\left(\mathscr{F}_{2}^{ \pm} H_{2} \varphi\right)(\zeta)=\omega(\zeta)\left(\mathscr{F}_{2}^{ \pm} \varphi\right)(\zeta)=\omega(\zeta)\left(\varphi, \phi_{2}^{ \pm}(\zeta)\right)
$$

shows (2.3.8).

Q.E.D.

This lemma shows that the functions $\phi_{2}^{ \pm}$obtained above can be regarded as eigenfunctions in a certain sense.

Remark 2.3.4. Put $e_{n}=(n, n+1]$ for $n=1,2,3, \cdots$ and $e_{n}=\left(\frac{1}{2-n}, \frac{1}{1-n}\right]$ for $n=0,-1,-2, \cdots$. Then,

$$
W_{j k}^{ \pm}((0, \infty))=s-\lim _{N \rightarrow \infty} \sum_{n=-N}^{+N} W_{j k}^{ \pm}\left(e_{n}\right)
$$

is well defined and Theorem 2.2.4 holds with $e=(0, \infty)$. Moreover, assume that Assumption 2.3.1 holds with $e=(0, \infty)$. Then Theorem 2.3.2 also holds with $e=(0, \infty)$. Indeed, put $\Omega_{n}=\omega^{-1}\left(e_{n}\right)$ and $J_{n}$ be the operator on $L_{2}(\Omega, d \rho)$ such that

$$
\left(J_{n} \phi\right)(\zeta)=\left\{\begin{array}{cl}
\phi(\zeta) & \zeta \in \Omega_{n} \\
0 & \zeta \notin \Omega_{n}
\end{array}\right.
$$

Then the above theorem shows that

$$
J_{n} \mathscr{F}_{2}^{ \pm}=J_{n} \mathscr{F}_{1} W_{12}^{ \pm}\left(e_{n}\right) .
$$

Noting (2.3.9) and $J_{n} \mathscr{F}_{1} W_{12}\left(e_{m}\right)=0(n \neq m)$, sum (2.3.10) from $n=-\infty$ to $+\infty$. Then we have (2.3.5) with $e=(0, \infty)$.

\section{§3. Schrödinger Operators}

3.1. Let us consider the Schrödinger operator

$$
L=-\Delta+V(x), x \in R^{n}(n \geqq 2),
$$

where $V(x)$ is a real valued bounded function such that $V(x) \rightarrow 0$ as $|x| \rightarrow \infty$. As is well known the following holds (see e.g. [11; Chap. V§5]). The operators $-\Delta$ and $L$ restricted on $C_{0}^{\infty}$ are essentially selfadjoint in $\mathscr{H}=L_{2}\left(R^{n}\right)$. Their unique selfadjoint extensions will be denoted by $H_{1}$ and $H_{2}$, respectively. Here $C_{0}^{\infty}$ denotes the totality of infinitely continuously differentiable functions with compact support. The essential spectra of $H_{1}$ and $H_{2}$ coincide with each other 
and are exactly the non negative real axis. Thus the spectrum of $H_{2}$ on the negative real axis consists only of isolated eigenvalues with finite multiplicities, having zero as its only possible limit point.

As to the nature of the positive spectrum of $\mathrm{H}_{2}$, we have Lemma 3.2.3 due to Ikebe and Saitō [9]. Before stating it, we shall introduce notations and assumption.

3.2. Let $\beta$ be a real number and $G$ be a domain in $R^{n}$. We denote by $L_{2, \beta}(G)$ the Hilbert space of all functions $f$ on $G$ such that $(1+|x|)^{\beta} f \in L_{2}(G)$. The norm is denoted by \|\|$_{\beta, G^{*}}$ We write $L_{2, \beta}\left(\mathbb{R}^{n}\right)=L_{2, \beta}$ and \|\|$_{\beta, R^{n}}=\|\|_{\beta}$ in short.

Assumption 3.2.1. We assume that $V(x)$ can be decomposed as the sum $V_{1}(x)+V_{2}(x)$ such that

(i) $V_{1}$ is a real valued $C^{1}$ function satisfying

$$
\left\{\begin{array}{l}
\left|V_{1}(x)\right| \leqq C(1+|x|)^{-\delta} \\
\left|\operatorname{grad} V_{1}(x)\right| \leqq C(1+|x|)^{-1-\delta} \quad(\delta>0)
\end{array}\right.
$$

and

(ii) $V_{2}$ is a real valued continuous function satisfying

$$
\left|V_{2}(x)\right| \leqq C(1+|x|)^{-1-\delta^{\prime}} \quad\left(\delta^{\prime}>0\right) .
$$

Lemma 3.2.2. Let Assumption 3.2.1 be satisfied. Then Assumption 2.2.1 is satisfied with $\mathcal{H}_{+}=L_{2,(1+\varepsilon) / 2}$ for sufficiently small $\varepsilon>0$. In particular $E_{j}((0, \infty)) H_{j}$ is absolutely continuous $(j=1,2)$.

This is due to Ikebe and Saitō [9; Theorems 1.4 and 1.5].

Let Assumption 3.2.1 be satisfied. We may assume without loss of generality that

$$
V_{1}(x)=0 \text { in }|x| \leqq 1
$$

$\delta<1$ and

$$
\left|\frac{\partial}{\partial x_{j}} \frac{\partial}{\partial x_{k}} V_{1}(x)\right| \leq C(1+|x|)^{-(1+2 \delta)+\varepsilon}
$$

for any $\varepsilon>0$. The last one follows from Lemma 3.3 of Hörmander [4].

We put

$$
X(k, x)=\frac{1}{2 k} \int_{0}^{r} V_{1}(s \tilde{x}) d s
$$


Here and in the sequel we use the notations that $r=|x|, \tilde{x}=x / r$ and $\tilde{x}_{j}$ is the $j$-th component of $\tilde{x}$. It is easy to see that $X(k, x)$ has the following properties:

$$
X(k, x)=0 \text { in }|x| \leqq 1
$$

and

$$
\left\{\begin{aligned}
\operatorname{grad} X(k, x) & =O\left(r^{-\delta}\right) \\
\Delta X(k, x) & =O\left(r^{-2 \delta+\varepsilon}\right)
\end{aligned}\right.
$$

as $r \rightarrow \infty$. Let $U(k)$ be the operator of multiplication by $\exp [-i X(k, x)]$, which is unitary in $L_{2, \beta}$ for any real $\beta$. We put

$$
U_{21}^{ \pm}(\lambda)=U( \pm \sqrt{\lambda})=\exp [-i X( \pm \sqrt{\lambda}, x)]
$$

and

$$
U_{12}^{ \pm}(\lambda)=U( \pm \sqrt{\lambda})^{*}=\exp [i X( \pm \sqrt{\lambda}, x)]
$$

for $\lambda>0$.

We owe the following lemma to Pinchuk [14; Theorem 5.1].

Lemma 3.2.3. Let Assumption 3.2 .1 be satisfied with $\delta, \delta^{\prime}>1 / 2$. Let $U_{21}^{ \pm}(\lambda)$ and $U_{12}^{ \pm}(\lambda)$ be as above. Then Assumption 2.2.3 holds with $\mathscr{H}_{+}=L_{2,(1+\varepsilon) / 2}$ and $\widetilde{\mathcal{H}}_{+}=L_{2,1+\varepsilon / 2}$ for sufficiently small $\varepsilon>0$.

(We will use this lemma only the case that $V_{2} \equiv 0$.)

3.3. At first let us consider the Fourier transformation $\left(\mathscr{F}^{\prime} f\right)(\zeta)=(2 \pi)^{-n / 2} \times$ $\int f(x) e^{-i(\zeta \cdot x)} d x, \zeta \in R^{n}$. Let $\Omega=R^{n}, \omega(\zeta)=|\zeta|^{2}$, do the usual Lebesque measure on $R^{n}, \phi_{1}(\zeta, \cdot)=(2 \pi)^{-n / 2} e^{+i(\zeta \cdot \cdot)}$ and $H_{1}=-\Delta$. Then Assumption 2.3.1 is satisfied with $\mathcal{H}_{+}=L_{2, \beta}$ for $\beta>n / 2$. On the other hand when we want to use Lemmas 2.3.1 and 2.3.2, we must take $\mathscr{H}_{+}=L_{2,(1+\varepsilon) / 2} \supseteqq L_{2, \beta}(\beta>n / 2, n \geqq 2)$. Therefore we give up to construct the eigenfunction expansion in terms of plane waves and will do it in terms of spherical waves.

Let $\left\{Y_{l m}(\theta)\right\}$ be a system of $(n-1)$-dimensional spherical harmonics which forms a complete orthonormal systems in $L_{2}\left(S^{n-1}\right)$. Here $l=0,1,2, \cdots$ and $m$ runs over a finite set which depends on $n$ and $l$. The function $Y_{l m}(\theta)$ is an eigenfunction of the Laplace-Beltrami operator $\Lambda$ on the unit sphere $S^{n-1}$ belonging to the eigenvalue $-l(l+n-2)$.

We put

$$
\phi_{1}(x ; k, l, m)=r^{-(n-2) / 2} J_{\nu}(k r) Y_{l m}(\tilde{x}),
$$

where $r=|x|, x=\tilde{x} / r, k>0$ and $J_{v}(z)$ is the Bessel function of order $\nu$ given by 


$$
\nu=\left[l(l+n-2)+\left(\frac{n-2}{2}\right)^{2}\right]^{1 / 2} .
$$

Then it satisfies the equation

$$
-\Delta \phi_{1}(x ; k, l, m)=k^{2} \phi_{1}(x ; k, l, m) .
$$

Let $\Omega$ be the set

$$
\Omega=\{\zeta=(k, l, m) ; k>0 \text { and } l, m \text { are as above }\},
$$

the measure $d \rho$ on $\Omega$ be the direct product of $k d k$ on $(0, \infty)$ and the point measure on $(l, m)$-set, and $\omega(\zeta)=k^{2}$. The well known formula

$$
J_{\nu}(r) \approx \sqrt{\frac{2}{\pi r}} \cos \left(r-\frac{(2 \nu+1)}{4} \pi\right) \text { as } r \rightarrow \infty
$$

implies

$$
\phi_{1}(x ; k, l, m)=O\left(r^{-(n-1) / 2}\right) \text { as } r \rightarrow \infty
$$

so that $\phi_{1} \in L_{2,-(1+\varepsilon) / 2}$. Thus as is well known it holds that

Lemma 3.3.1 The Assumption 2.3.1 is satisfied with $\phi_{1}$ defined as above, $e=(0, \infty)$ and $\mathcal{H}_{+}=L_{2,(1+\varepsilon) / 2}$.

\section{§4. Eigenfunction Expansion Theorem}

4.1. The aim of this section is to prove the following theorem. We note that the condition (4.1.3) is a modified version of (1.5).

Theorem 4.1.1. Let Assumption 3.2.1 be satisfied for $\delta>1 / 2$ and $\delta^{\prime}>0$. Then the equation

$$
\left(L-k^{2}\right) \phi=0
$$

has the unique solution in the distribution sence such that

$$
u=\phi-U( \pm k) \phi_{1}(\cdot ; k, l, m)
$$

satisfies the condition

$$
\left\{\begin{array}{l}
u \in L_{2,-(1+\varepsilon) / 2} \\
\left(\operatorname{grad}+\frac{n-1}{2 r} \tilde{x} \pm i k \tilde{x}\right) u \in L_{2,(-1+\varepsilon) / 2}
\end{array}\right.
$$

for each sign and each $(k, l, m) \in \Omega$. We denote this unique solution by $\phi_{2}^{ \pm}(x ; k, l, m)$. 
Let $\Omega$ and $d \rho$ be the same as those in Section 3.3. Define

$$
\left(\mathscr{L}_{2}^{ \pm} f\right)(k, l, m)=1 . \mathrm{i} . \mathrm{m} \cdot \int_{|x| \leqq R} f(x) \overline{\phi_{2}^{ \pm}(x ; k, l, m)} d x
$$

for $f \in L_{2}\left(R^{n}\right)$, where 1.i.m. means the limit in $L_{2}(\Omega, d \rho)$. Then $\mathscr{F} \frac{ \pm}{2}$ is a unitary operator from $L_{2}\left(R^{n}\right)$ onto $L_{2}(\Omega, d \rho)$ and it holds that

$$
\left(\mathscr{F}_{2}^{+} E_{2}(\Delta) f\right)(k, l, m)=\chi_{\Delta}\left(k^{2}\right)\left(\mathscr{F}_{2} f\right)(k, l, m) \quad d \rho \text {-a.e. }
$$

for any $f \in L_{2}\left(R^{n}\right)$ and any Borel set $\Delta \subset(0, \infty)$.

Proof. Let $w$ be the difference of two solutions of the equation (4.1.1) satisfying the condition (4.1.3). Then it is a solution of the same equation satisfying the condition (4.1.3) with $u$ replaced by $w$. Therefore $w=0$ by virtue of Lemma 1.9 (ii) of Ikebe and Saitō [9], which proves the uniqueness.

Let $H_{3}$ be $H_{2}$ with $V_{2}=0$. Then Lemmas 3.2.2, 3.2.3, 3.3.1 and Theorem 2.3.2 implies that

$$
\phi_{3}^{ \pm}(k, l, m)=G_{13}^{\dagger}\left(k^{2} \pm i 0\right) \phi_{1}(\cdot ; k, l, m)
$$

gives the two systems of eigenfunctions in the sense stated in Section 2.3, where $U_{13}^{+}=U_{12}^{ \pm}$and $U_{31}^{+}=U_{21}^{ \pm}$. Let $\mathscr{D}=C_{0}^{\infty}$. Then the assumptions of Lemma 2.3.3 are satisfied so that the equation (2.3.8) holds, which implies that $\phi_{3}^{+}$satisfies (4.1.1) with $V_{2} \equiv 0$ in the distribution sense. Now let us assume that $u$ defined by

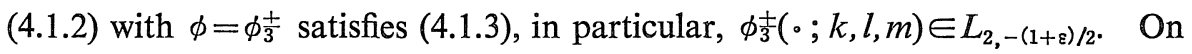
the other hand Lemma 3.2.2 and the assumption on $V_{2}$ implies that

$$
\begin{aligned}
G_{23}(\lambda \pm i \mu) & =\left[H_{2}-(\lambda \pm i \mu)\right] R_{3}(\lambda \pm i \mu) \\
& =I+V_{2} R_{3}(\lambda \pm i \mu)
\end{aligned}
$$

and

$$
\begin{aligned}
G_{32}(\lambda \pm i \mu) & =\left[H_{3}-(\lambda \pm i \mu)\right] R_{2}(\lambda \pm i \mu) \\
& =I-V_{2} R_{2}(\lambda \pm i \mu)
\end{aligned}
$$

satisfy Assumption 2.2.3 with the suffix 1 replaced by $3, U_{23}=U_{32}=$ identity and $\mathscr{H}_{+}=\tilde{\mathscr{H}}_{+}=L_{2,(1+\varepsilon) / 2}$. Now we have

$$
\begin{aligned}
\phi_{2}^{ \pm}(\cdot ; k, l, m) & =G_{12}\left(k^{2} \pm i 0\right)^{\dagger} \phi_{1}(\cdot ; k, l, m) \\
& =\left[G_{13}\left(k^{2} \pm i 0\right) G_{32}\left(k^{2} \pm i 0\right)\right]^{\dagger} \phi_{1}(\cdot ; k, l, m) \\
& =G_{32}\left(k^{2} \pm i 0\right)^{\dagger} \phi_{3}^{ \pm}(\cdot ; k, l, m) \\
& =\phi_{3}^{ \pm}(\cdot ; k, l, m)-R_{2}\left(k^{2} \mp i 0\right) V_{2} \phi_{3}^{ \pm}(\cdot ; k, l, m),
\end{aligned}
$$


which satisfies (4.1.1) in the distribution sense by virtue of the same argument as that on $\phi_{3}$. Now $R_{2}\left(k^{2} \mp i 0\right) V_{2} \phi_{3}$ satisfies the radiation condition (4.1.3) by virtue of Theorem 1.4 of [9]. Thus we know that $\phi_{3}^{+}-U \phi_{1}$ and $\phi_{2}^{+}-\phi_{3}^{+}$satisfy (4.1.3) so that their sum $\phi_{2}^{ \pm}(\cdot ; k, l, m)-U( \pm k) \phi_{1}(\cdot ; k, l, m)$ also satisfies (4.1.3). We define

$$
\left(\mathscr{F}_{2}^{\frac{1}{2}} f\right)(k, l, m)=\left(f, \phi_{2}^{\frac{1}{2}}(\cdot ; k, l, m)\right)
$$

for $f \in L_{2,(1+\varepsilon) / 2}$. Then Theorem 2.3.2 shows that this operator $\mathscr{F}_{2} \frac{ \pm}{2}$ can be uniquely extended to the unitary operator from $L_{2}\left(R^{n}\right)$ onto $L_{2}(\Omega, d \rho)$ and this extension satisfies (4.1.5). The operators defined by (4.1.4) and (4.1.4) ${ }^{\prime}$ are coincide with each other for $f \in L_{2}$ with compact support so that the definition (4.1.4) is well defined and this $\mathscr{F}_{2}^{\frac{ \pm}{2}}$ has the properties stated in the present theorem.

In conclusion, we will complete the proof of the present theorem if we show the following lemma.

Lemma 4.1.2. Define $\phi_{3}^{\frac{t}{3}}(\cdot ; k, l, m)$ by (4.1.6) and $u$ by (4.1.2) with $\phi=\phi_{3}^{ \pm}$. Then $u$ satisfies (4.1.3).

This lemma will be proved at the end of Section 4.3.

4.2. Now we must show Lemma 4.1.2. We will show it only for $\phi_{3}^{+}$. (The proof for $\phi_{3}^{-}$is similar.) So we omit the sufficies and put

$$
\begin{aligned}
\phi(\mu) & =\phi(\cdot ; k, l, m ; \mu) \\
& =G_{13}^{\dagger}\left(k^{2}+i \mu\right) \phi_{1}(\cdot ; k, l, m) \quad(\mu \geqq 0)
\end{aligned}
$$

and

$$
\begin{aligned}
u(\mu) & =u(\cdot ; k, l, m ; \mu) \\
& =\phi(\cdot ; k, l, m ; \mu)-U(k) \phi_{1}(\cdot ; k, l, m) \quad(\mu \geqq 0),
\end{aligned}
$$

where $U(k)=\exp [-i X(k, \circ)]$ and $X(k, x)$ is defined by (3.2.5). We put

$$
\kappa=\sqrt{k^{2}-i \mu}=-\kappa_{1}+i \kappa_{2} \quad\left(\kappa_{1}>0, \kappa_{2} \geqq 0\right)
$$

and

$$
\left\{\begin{array}{l}
\mathscr{D}_{j} u=\mathscr{D}_{j}^{(\kappa)} u=\left(\partial / \partial x_{j}+(n-1) \tilde{x}_{j} /(2 r)-i \kappa \tilde{x}_{j}\right) u \\
\mathscr{D} u=\left(\mathscr{D}_{1} u, \mathscr{D}_{2} u, \cdots, \mathscr{D}_{n} u\right) \\
\mathscr{D}_{r} u=\sum_{j=1}^{n} \tilde{x}_{j} \mathscr{D}_{j} u=(\partial / \partial r+(n-1) /(2 r)-i \kappa) u,
\end{array}\right.
$$

where $r=|x|, \tilde{x}_{j}=x_{j} / r$ and $\tilde{x}=\left(\tilde{x}_{1}, \tilde{x}_{2}, \cdots, \tilde{x}_{n}\right)$. 
In the sequel we fix $(k, l, m) \in \Omega$ and assume that Assumption 3.2.1 is satisfied with $\delta>\frac{1}{2}$ and $V_{2} \equiv 0$.

Lemma 4.2.1 (1) $\phi(\mu) \rightarrow \phi(0)$ weakly in $L_{2,-(1+\varepsilon / 2)}$ as $\mu \downarrow 0$.

(2) $\phi(\mu)=\phi(\cdot ; k, l, m ; \mu)(\mu \geqq 0)$ satisfies the equation

$$
\left(L-\kappa^{2}\right) \phi(\mu)=i \mu U(k) \phi_{1}(x ; k, l, m)
$$

in the distrubution sense.



$$
\left(L-\kappa^{2}\right) u(\mu)=f=f_{1}+f_{2}
$$

in the distrubution sense, where

$$
f_{1}=-2 i U(k) \frac{\partial X}{\partial r}(k, x)\left(\partial / \partial r+\frac{n+1}{2 r}-i k\right) \phi_{1}(x ; k, l, m)
$$

and

$$
\begin{aligned}
f_{2}= & -2 i U(k) \sum_{j} \frac{\partial X}{\partial x_{j}}(k, x)\left(\frac{\partial}{\partial x_{j}}-\tilde{x}_{j} \frac{\partial}{\partial r}\right) \phi_{1}(x ; k, l, m) \\
& -U(k)\left\{i \Delta X-i \frac{n-1}{r} \frac{\partial X}{\partial r}+|\operatorname{grad} X|^{2}\right\} \phi_{1}(x ; k, l, m) .
\end{aligned}
$$

Moreover, we have

$$
\left\{\begin{array}{l}
f_{1} \in L_{2, \delta-(1+\varepsilon) / 2} \\
f_{2} \in L_{2,2 \delta-(1+\varepsilon) / 2} \subset L_{2,(1+\varepsilon) / 2} .
\end{array}\right.
$$

Proof. The assertion (1) follows immediately from (3) in Assumption 2.2.3 with $\mathscr{H}_{+}=L_{2,(1+\varepsilon) / 2}$ and $\widetilde{\mathscr{H}}_{+}=L_{2,1+\varepsilon / 2}$, which is guaranteed by Lemma 3.2.3.

Let $\psi$ be in $\tilde{\mathscr{H}}_{+}=L_{2,1+\varepsilon / 2}$ and $\mu>0$. Then

$$
\begin{aligned}
& (\phi(\mu), \psi)=\left(\phi_{1}, G_{13}\left(k^{2}+i \mu\right) \psi\right) \\
= & \left(\phi_{1},\left[H_{1}-\left(k^{2}+i \mu\right)\right] U^{*}(k)\left[H_{3}-\left(k^{2}+i \mu\right)\right]^{-1} \psi\right) .
\end{aligned}
$$

Put $\psi=\left[L-\left(k^{2}+i \mu\right)\right] \varphi, \varphi \in C_{0}^{\infty}$. Then we have

$$
\begin{aligned}
& \left(\phi(\mu),\left[L-\left(k^{2}+i \mu\right)\right] \varphi\right) \\
= & \left(\phi_{1},\left[H_{1}-\left(k^{2}+i \mu\right) U^{*}(k) \varphi\right)=i \mu\left(U(k) \phi_{1}, \varphi\right)\right.
\end{aligned}
$$

for any $\varphi \in C_{0}^{\infty}$, which implies (4.2.5) for $\mu>0$. Let $\mu \downarrow 0$ in the above equation to obtain (4.2.6) for $\mu=0$. Thus we have the assertion (2).

A direct computation shows that 


$$
\left(L-\kappa^{2}\right)\left(U(k) \phi_{1}\right)=-f+i \mu U(k) \phi_{1},
$$

where

$$
\begin{aligned}
f= & -U(k)\left[2 i(\operatorname{grad} X) \cdot\left(\operatorname{grad} \phi_{1}\right)\right. \\
& \left.+\left(i \Delta X+|\operatorname{grad} X|^{2}+V\right) \phi_{1}\right] .
\end{aligned}
$$

Subtraction (4.2.10) from (4.2.9) yields $\left(L-\kappa^{2}\right) u(\mu)=f$. Noting the definition (3.2.5), it is easy to see that $f$ defined by (4.2.11) can be decomposed as the sum of $f_{1}$ and $f_{2}$ defined by (4.2.7) and (4.2.8), respectively.

Now the estimates

$$
\phi_{1}, \frac{\partial \phi_{1}}{\partial r} \text { and } r\left(\frac{\partial}{\partial x_{j}}-\tilde{x}_{j} \frac{\partial}{\partial r}\right) \phi_{1}=O\left(r^{-(n-1) / 2}\right)
$$

and (3.2.7) show (4.2.9). The first one in (4.2.12) is nothing but (3.3.5), the second follows from (3.3.4) and the well known formula $J_{\nu}^{\prime}(r)=r^{-1} J_{\nu}(r)-J_{\nu+1}(r)$, and the third follows from the facts that $\left(\frac{\partial}{\partial x_{j}}-\tilde{x}_{j} \frac{\partial}{\partial r}\right) \phi_{1}(x ; k, l, m)=r^{-(n-2) / 2} \times$ $J_{\nu}(k r)\left(\frac{\partial}{\partial x_{j}}-\tilde{x}_{j} \frac{\partial}{\partial r}\right) Y_{l m}(\tilde{x})$ and that $r\left(\frac{\partial}{\partial x_{j}}-\tilde{x}_{j} \frac{\partial}{\partial r}\right) \psi$ is bounded in $r \geqq 1$ for any smooth function $\psi$ depending only on $\tilde{x}$.

Q.E.D.

If $f$ is in $L_{2,(1+\varepsilon) / 2}$, then the limiting absorption principle (Theorem 1.4 of [9]) shows that $u(\mu)$ converges to some $u$ in $L_{2,-(1+\varepsilon) / 2}$, which coincides with $u(0)$ by virtue of (1) of Lemma 4.2.1 and it satisfies (4.1.3) with upper sign. But in our case $f \notin L_{2,(1+\varepsilon) / 2}$ in general so that we must follow the calculations in [9] step by step.

4.3. We put $E_{r}=\{x ;|x| \geqq r\}$. The following two lemmas will be shown in the next section.

Lemma 4.3.1 Let $\kappa=-\kappa_{1}+i \kappa_{2}, \kappa_{2}>0$ and $\beta$ be real. Let $v \in L_{2, \beta}$ satisfy the equation

$$
\left(L-\kappa^{2}\right) v=f
$$

in the distribution sense, where $f=f_{1}+f_{2}$ and $f_{1}$ and $f_{2}$ are defined by (4.2.7) and (4.2.8), respectively. Then we have

$$
v \in L_{2,-(1+q) / 2} \cap H_{2, l o c}
$$

and

$$
\|\mathscr{D} v\|_{(-1+\varepsilon) / 2, E_{1}} \leqq C\left\{\|v\|_{-(1+\varepsilon) / 2}+1\right\}
$$

for sufficiently small $\varepsilon$. Here and in the sequel $C$ denotes several constants not 
depending on $\kappa_{2}>0$.

Lemma 4.3.2. Let $v$ be as in Lemma 4.3.1. Then there is a constant $C$ not depending on $\rho, \kappa_{2}$ and $v$ such that

$$
\|v\|_{-(1+\varepsilon) / 2, E_{\rho}} \leqq C \rho^{-\varepsilon}\|\mathscr{D} v\|_{(-1+\varepsilon) / 2, E_{1}}+\alpha(\rho)(\rho \geqq 1),
$$

where $\alpha$ is a certain function not depending on $v$ and $\kappa_{2}$ and having the property that $\alpha(\rho) \rightarrow 0$ as $\rho \rightarrow \infty$.

The following lemma is essentially Lemma 1.11 of [9] so we omit the proof.

Lemma 4.3.3. Let $\left\{v_{m}\right\}$ be a sequence in $L_{2,-(1+\varepsilon) / 2}$ and $\mu_{m} \downarrow 0$. Assume that

$$
\left\{\begin{array}{l}
g_{m} \equiv\left(L-\left(k^{2}-i \mu_{m}\right)\right) v_{m} \in L_{2, \beta} \\
g_{m} \rightarrow g \text { in } L_{2, \beta} \text { as } m \rightarrow \infty
\end{array}\right.
$$

with some real $\beta$, and there is a constant $C$ not depending on $m$ such that

$$
\left\{\begin{array}{l}
\left\|v_{m}\right\|_{-(1+\varepsilon) / 2} \leqq C \\
\left\|\mathscr{D}^{(m)} v_{m}\right\|_{(-1+\varepsilon) / 2, E_{1}} \leqq C \\
\left\|v_{m}\right\|_{-(1+\varepsilon) / 2, E_{\rho}} \rightarrow 0 \text { as } \rho \rightarrow \infty \quad \text { uniformly in } m,
\end{array}\right.
$$

where $\mathscr{D}^{(m)}$ is defined by (4.2.4) with $\kappa=\sqrt{k^{2}-i \mu_{m}}$.

Then $\left\{v_{m}\right\}$ has a strong limit $v$ in $L_{2,-(1+\varepsilon) / 2}$, which satisfies

$$
\begin{gathered}
\left(L-k^{2}\right) v=g \\
\left\|\mathscr{D}^{(-k)} v\right\|_{(-1+\varepsilon) / 2, E_{1}}<+\infty
\end{gathered}
$$

and

$$
\mathscr{D}^{(m)} v_{m} \rightarrow \mathscr{D}^{(-k)} v \text { in } L_{2}\left(E_{1}\right)_{l o c} \text { as } m \rightarrow \infty .
$$

Lemma 4.3.4. Let $u(\mu)(\mu>0)$ be as in Section 4.2. Then $u(\mu)$ satisfies the following:

(1) $\|u(\mu)\|_{-(1+\varepsilon) / 2} \leqq C$.

(2) $\left\|\mathscr{D}^{(\kappa)} u(\mu)\right\|_{(-1+\varepsilon) / 2} \leqq C \quad\left(\kappa=\sqrt{\left.k^{2}-i \mu\right)}\right.$.

(3) $\|u(\mu)\|_{-(1+\varepsilon) / 2, E_{p}} \rightarrow 0$ as $\rho \rightarrow \infty$ uniformly in $\mu$.

Proof. Lemma 4.2.1 shows that $u(\mu)$ satisfies the assumption on $v$ in Lemma 4.3.1 with $\beta=-(1+\varepsilon / 2)$. Thus Lemmas 4.3.1 and 4.3.2 show that $u(\mu) \in L_{2,-(1+\varepsilon) / 2}$ and that (1) implies (2) and (3). Therefore, it is sufficient to show (1) alone. Let us assume that (1) is false. Then there is a sequence $\left\{\mu_{m}\right\}$ such that $\mu_{m} \downarrow 0$ as $m \rightarrow \infty$ and $\alpha_{m}^{-1} \equiv\left\|u\left(\mu_{m}\right)\right\|_{-(1+\varepsilon) / 2} \rightarrow \infty$. Then $v_{m} \equiv \alpha_{m} u\left(\mu_{m}\right)$ 
satisfies (4.3.5) with $g_{m}=\alpha_{m} f$ and $g=0$ by virtue of (4.2.6) and (4.2.9). The assertion (4.3.6) follows from $\left\|v_{m}\right\|_{-(1+\varepsilon) / 2}=1$ and Lemmas 4.3.1 and 4.3.2. Therefore, we can apply Lemma 4.3 .3 to see that $\left\{v_{m}\right\}$ has a strong limit $v$ in $L_{2,-(1+\varepsilon) / 2}$ which satisfies (4.3.7) with $g=0$ and (4.3.8). Therefore, we have $v=0$ by virtue of Lemma 1.9(ii) of [9]. But this is a contradiction because $\|v\|_{-(1+\varepsilon) / 2}$ $=1$ by virtue of $\left\|v_{m}\right\|_{-(1+\varepsilon) / 2}=1$.

Q.E.D.

Proof of Lemma 4.1.2. Lemma 4.3.4 shows that we can apply Lemma 4.3.3 to obtain that $u(\mu)$ has a strong limit $u$ in $L_{2,-(1+\varepsilon) / 2}$ as $\mu \downarrow 0$ which satisfies (4.3.7) with $g$ replaced by $f$ and (4.3.8). On the other hand Lemma 4.2.1 (1) shows that this $u$ coincides with $u(0)$ defined by (4.2.2) with $\mu=0$, that is, $u$ defined by (4.1.2).

Q.E.D

\section{§5. Proof of Lemmas 4.3 .1 and 4.3 .2}

5.1. We put $E_{r}=\{x ;|x| \geqq r\}, B_{r}=\{x ;|x| \leqq r\}, B_{r s}=\{x ; r \leqq|x| \leqq s\}$ and $S_{r}=\{x ;|x|=r\}$.

Lemma 5.1.1. Let $v \in H_{1, l o c}\left(E_{1}\right) \cap L_{2}$. Then

$$
\lim _{R \rightarrow \infty} \int_{S_{R}} R|v|^{2} d S=0
$$

and

$$
\varliminf_{R \rightarrow \infty} \frac{d}{d R} \int_{S_{R}}|v|^{2} d S \leqq 0
$$

Proof. The surface integrals have a meaning for $R>1$ since $v \in H_{1, l o c}\left(E_{1}\right)$. If (5.1.1) is not true, then there is a constant $d>0$ such that $\int_{S_{R}}|v|^{2} d S \geqq d / R$ for large $R$, which contradicts the assumption $v \in L_{2}$. If (5.1.2) is not true, then there is a constant $d>0$ such that $\frac{d}{d R} \int_{S_{R}}|v|^{2} d S \geqq d$ so that $\int_{S_{R}}|v|^{2} d S \geqq d R+c$ for large $R$, which contradicts the assumption $v \in L_{2}$.

Q.E.D.

Lemma 5.1.2. (1) Let $\kappa$ be a complex number and $\beta$ be real. Let $v \in$ $L_{2, \beta} \cap H_{2, l o c}$ satisfy

$$
\left(L-\kappa^{2}\right) v=g \in L_{2, \beta}
$$

Then we have grad $v \in L_{2, \beta}$.

(2) Let $\kappa=\kappa_{1}+i \kappa_{2}, \kappa_{1} \kappa_{2} \neq 0$ and $\beta$ be real. Let $v \in H_{2, l o c}$ satisfy $\left(L-\kappa^{2}\right) v=$ $g \in L_{2, \beta}$ and $v, \operatorname{grad} v \in L_{2, \beta-1 / 2}$. Then $v \in L_{2, \beta}$ and 


$$
\left|\kappa_{1} \kappa_{2}\right|\|v\|_{\beta} \leqq C\left\{\left\|\mathscr{D}^{(\kappa)} v\right\|_{\beta-1, E_{2}}+\|v\|_{\beta-1}+\|g\|_{\beta}\right\} .
$$

Proof. (1) Let $\varphi$ be a real valued smooth function depending only on $r=$ $|x|$. Multiply both sides of (5.1.3) by $\varphi \bar{v}$, integrate (by parts) over $B_{R}$. Then we have

$$
\begin{aligned}
& \quad \int_{B_{R}} \varphi|\operatorname{grad} v|^{2} d x+\int_{B_{R}} \frac{\partial \varphi}{\partial r} \frac{\partial v}{\partial r} \bar{v} d x-\int_{S_{R}} \varphi \frac{\partial v}{\partial r} \bar{v} d S \\
& +\int_{B_{R}} \varphi\left(V-\kappa^{2}\right)|v|^{2} d x=\int_{B_{R}} \varphi g \bar{v} d x .
\end{aligned}
$$

Put $\varphi=(1+r)^{2 \beta}$. The second term of (5.1.5) is estimated by

$$
2 \beta\|v\|_{\beta}\left\{\int_{B_{R}}(1+r)^{2 \beta}|\operatorname{grad} v|^{2} d x\right\}^{1 / 2}
$$

so that, taking the real part of (5.1.5), we have

$$
\int_{B_{R}} \varphi|\operatorname{grad} v|^{2} d x \leqq C\left\{\operatorname{Re} \int_{S_{R}} \varphi \frac{\partial v}{\partial r} \bar{v} d S+\|v\|_{\beta}^{2}+\|g\|_{\beta}^{2}\right\} .
$$

In order to prove the present lemma, it is sufficient to show that

$$
\varliminf_{\bar{R} \rightarrow \infty} \operatorname{Re} \int_{S_{R}} \varphi \frac{\partial v}{\partial r} \bar{v} d S \leqq 0
$$

Now, the integration by parts yields

$$
2 \operatorname{Re} \int_{B_{R}} \varphi \frac{\partial v}{\partial r} \bar{v} d x=\int_{S_{R}} \varphi|v|^{2} d S-\int_{B_{R}}\left(\varphi \frac{n-1}{r}+\frac{\partial \varphi}{\partial r}\right)|v|^{2} d x .
$$

Differentiate both sides of this equation with respect to $R$ to obtain

$$
\frac{1}{2} \frac{d}{d R} \int_{S_{R}} \varphi|v|^{2} d S=\operatorname{Re} \int_{S_{R}} \varphi \frac{\partial v}{\partial r} \bar{v} d S+\frac{1}{2} \int_{S_{R}}\left(\varphi \frac{n-1}{R}+\frac{\partial \varphi}{\partial r}\right)|v|^{2} d S .
$$

Thus Lemma 5.1.1 shows (5.1.6), which proves (1).

(2) Taking the imaginary part of (5.1.5), we have

$$
\begin{aligned}
& \operatorname{Im} \int_{B_{R}} \frac{\partial \varphi}{\partial r} \frac{\partial v}{\partial r} \bar{v} d x-\operatorname{Im} \int_{S_{R}} \varphi \frac{\partial v}{\partial r} \bar{v} d S+2 \kappa_{1} \kappa_{2} \int_{B_{R}} \varphi|v|^{2} d x \\
= & \operatorname{Im} \int_{B_{R}} \varphi g \bar{v} d x,
\end{aligned}
$$

and so we have

$$
2\left|\kappa_{1} \kappa_{2}\right| \int_{B_{R}} \varphi|v|^{2} d x \leqq \int_{S_{R}} \varphi\left|\frac{\partial v}{\partial r}\right||v| d S+
$$




$$
\begin{aligned}
& +\left[\int_{B_{R}} \varphi|v|^{2} d x\right]^{1 / 2} \times\left[\left(\int_{B_{R}} \varphi^{-1}\left(\frac{\partial \varphi}{\partial r}\right)^{2}\left|\mathcal{D}_{r}^{(\kappa)} v\right|^{2} d x\right)^{1 / 2}\right. \\
& \left.+\left|\kappa_{1}\right|\left(\int_{B_{R}} \varphi^{-1}\left|\frac{\partial \varphi}{\partial r}\right|^{2}|v|^{2} d x\right)^{1 / 2}+\left(\int_{B_{R}} \varphi|g|^{2} d x\right)^{1 / 2}\right] .
\end{aligned}
$$

Let $\varphi=(1+r)^{2 \beta}$ in $r \geqq 3$ and $=1$ in $r \leqq 2$. Devide both sides of this inequality by $\left(\int_{B_{R}} \varphi|v|^{2} d x\right)^{1 / 2}$ to obtain

$$
\begin{gathered}
\left|\kappa_{1} \kappa_{2}\right|\|v\|_{\beta_{,} B_{R}} \leqq C\left[\int_{S_{R}} \varphi\left|\frac{\partial v}{\partial r}\right||v| d S /\|v\|_{\beta, B_{R}}\right. \\
\left.+\left\|\mathscr{D}_{r}^{(\kappa)} v\right\|_{\beta-1, E_{2}}+\|v\|_{\beta-1}+\|g\|_{\beta}\right] .
\end{gathered}
$$

Let $R$ tend to $\infty$. Then the assumptions that $v, \operatorname{grad} v \in L_{2, \beta-1 / 2}$ and Lemma 5.1.1 show that

$$
\varliminf_{R \rightarrow \infty} \int_{S_{R}} \varphi\left|\frac{\partial v}{\partial r}\right||v| d S=0
$$

which yields the estimate (5.1.4).

Q.E.D.

Now we can prove the first half of Lemma 4.3.1.

Corollary 5.1.3. Let $v$ be as in Lemma 4.3.1 and $\varepsilon$ be sufficiently small. Then we have

$$
\left\{\begin{array}{l}
v \in L_{2, \delta-(1+\varepsilon) / 2} \cap H_{2, l o c} \subset L_{2, \mathrm{e} / 2} \\
\mathscr{D} v \in L_{2, \mathrm{e} / 2}\left(E_{1}\right)
\end{array}\right.
$$

and

$$
\kappa_{2}\|v\|_{\delta-(1+\varepsilon) / 2} \leqq C\left\{\|\mathscr{D} v\|_{(-1+\varepsilon) / 2, E_{2}}+\|v\|_{-(1+\varepsilon) / 2}+1\right\} .
$$

Proof. Since $v \in L_{2, \beta}$ satisfies (4.3.1) and $v, \Delta v \in L_{2, l o c}$ so that $v \in H_{2, l o c}$. We can apply the above lemma, repeatedly, to obtain (5.1.8) and

$$
\kappa_{2}\|v\|_{\delta-(1+\varepsilon) / 2} \leqq C\left\{\|\mathscr{D} v\|_{\delta-(3+\varepsilon) / 2, E_{2}}+\|v\|_{\delta-(3+\varepsilon) / 2}+1\right\}
$$

since $f \in L_{2,8-(1+\varepsilon) / 2}$, which implies (5.1.9).

\section{2.}

Lemma 5.2.1. Let $v$ be as in Lemma 4.3.1. Let $\varphi$ be a real valued smooth function depending only on $r$ and vanishes in $r \leqq 1$. Then we have 


$$
\begin{aligned}
& \int_{B_{R}}\left\{\left(\kappa_{2} \varphi+\frac{1}{2} \frac{\partial \varphi}{\partial r}\right)|\mathscr{D} v|^{2}+\left(\frac{\varphi}{r}-\frac{\partial \varphi}{\partial r}\right)\left(|\mathscr{D} v|^{2}-\left|\mathscr{D}_{r} v\right|^{2}\right)\right\} d x \\
= & \int_{S_{R}} \varphi\left|\mathscr{D}_{r} v\right|^{2} d S-\frac{1}{2} \int_{S_{R}} \varphi|\mathscr{D} v|^{2} d S-\frac{1}{2} \int_{S_{R}} \varphi V_{1}|v|^{2} d S \\
& +\int_{B_{R}}\left\{\frac{1}{2}\left(\frac{\partial \varphi}{\partial r} V_{1}+\frac{\partial V_{1}}{\partial r} \varphi\right)-\kappa_{2} \varphi V_{1}\right\}|v|^{2} d x \\
& -\operatorname{Re} \int_{B_{R}} \varphi \frac{(n-1)(n-3)}{4 r^{2}} v \overline{\mathscr{D}_{r} v} d x \\
& +\operatorname{Re} \int_{B_{R}} \varphi f_{2} \overline{\mathscr{D}_{r} v} d x+\operatorname{Re} \int_{B_{R}} \varphi f_{1} \overline{\mathscr{D}_{r} v} d x .
\end{aligned}
$$

Sketch of the proof. Rewrite the equation (4.3.1) in the form

$$
-\sum_{j=1}^{n} \frac{\partial}{\partial x_{j}} \mathscr{D}_{j} v+\frac{n-1}{2 r} \mathscr{D}_{r} v+\left(\kappa_{2}+i \kappa_{1}\right) \mathscr{D}_{r} v+\left(V_{1}+\frac{(n-1)(n-3)}{4 r^{2}}\right) v=f,
$$

multiply both side of this equation by $\varphi \overline{\mathscr{D}_{r} v}$, integrate by parts over $B_{R}$ and take the real part. Then we have (5.2.1). For the details, see the proof of Lemma 2.2. of [9], only noting that the surface integrals appearing by integration by parts do not vanish in our case.

Q.E.D.

Let us calculate the last term of (5.2.1).

Lemma 5.2.2. Let $v$ be as above. Then we have

$$
\begin{aligned}
\int_{B_{R}} \varphi f_{1} \overline{D_{r} v} d x=\int_{S_{R}} \varphi f_{1} \bar{v} d S \\
+2 i \int_{B_{R}}\left\{\frac{\partial \varphi}{\partial r} U(k) \frac{\partial X}{\partial r}(k, x)-i \varphi U(k)\left(\frac{\partial X}{\partial r}(k, x)\right)^{2}+\varphi U(k) \frac{\partial^{2} X}{\partial r^{2}}\right\} \\
\quad \times\left(\frac{\partial}{\partial r}+\frac{n-1}{2 r}-i k\right) \phi_{1}(x ; k, l, m) \bar{v} d x \\
+2 i \int_{B_{R}} \varphi U(k) \frac{\partial X}{\partial r}(k, x)\left\{l(l+n-2)+\frac{(n-1)(n-3)}{4}\right\} r^{-2} \phi_{1} \bar{v} d x \\
+\left(\kappa_{2}+i\left(k-\kappa_{1}\right)\right) \int_{B_{R}} \varphi f_{1} \bar{v} d x .
\end{aligned}
$$

Proof. Recall the definition (4.2.7) of $f_{1}$. The integration by parts yields

$$
\begin{aligned}
& \int_{B_{R}} \varphi f_{1} \overline{D_{r} v} d x=\int_{S_{R}} \varphi f_{1} \bar{v} d S \\
& \quad+2 i \int_{B_{R}}\left\{\frac{\partial}{\partial r}\left(\varphi U \frac{\partial X}{\partial r}\right)\right\}\left(\frac{\partial}{\partial r}+\frac{n-1}{2 r}-i k\right) \phi_{1} \bar{v} d x \\
& \quad+2 i \int_{B_{R}} \varphi U \frac{\partial X}{\partial r}\left(\frac{\partial}{\partial r}+\frac{n-1}{2 r}-i \bar{\kappa}\right)\left(\frac{\partial}{\partial r}+\frac{n-1}{2 r}-i k\right) \phi_{1} \bar{v} d x .
\end{aligned}
$$


Now, we have

$$
\begin{aligned}
& \left(\frac{\partial}{\partial r}+\frac{n-1}{2 r}-i \bar{\kappa}\right)\left(\frac{\partial}{\partial r}+\frac{n-1}{2 r}-i k\right) \phi_{1}(x ; k, l, m) \\
= & \left(\frac{\partial}{\partial r}+\frac{n-1}{2 r}+i k\right)\left(\frac{\partial}{\partial r}+\frac{n-1}{2 r}-i k\right) \phi_{1}-i(\bar{\kappa}+k)\left(\frac{\partial}{\partial r}+\frac{n-1}{2 r}-i k\right) \phi_{1} \\
= & \left(\frac{\partial^{2}}{\partial r^{2}}+\frac{n-1}{r} \frac{\partial}{\partial r}+\frac{(n-1)(n-3)}{4 r^{2}}+k^{2}\right) \phi_{1}-i(\bar{\kappa}+k)\left(\frac{\partial}{\partial r}+\frac{n-1}{2 r}-i k\right) \phi_{1} \\
= & \left(\Delta+k^{2}-\frac{1}{r^{2}} \Lambda+\frac{(n-1)(n-3)}{4 r^{2}}\right) \phi_{1}-i(\bar{\kappa}+k)\left(\frac{\partial}{\partial r}+\frac{n-1}{2 r}-i k\right) \phi_{1} \\
= & \frac{1}{r^{2}}\left[l(l+n-2)+\frac{(n-1)(n-3)}{4}\right] \phi_{1}-\left(\kappa_{2}+i\left(k-\kappa_{1}\right)\right)\left(\frac{\partial}{\partial r}+\frac{n-1}{2 r}-i k\right) \phi_{1} .
\end{aligned}
$$

Put this equation into the last term of (5.2.3) to obtain (5.2.2).

Proof of Lemma 4.3.1. The assertion (4.3.2) has been already proved in Corollary 5.1.2.

Let us put $\varphi=\alpha(|x|)(1+|x|)^{\varepsilon}$, where $\alpha(r)$ is a smooth function such that $0<\alpha<1, \alpha^{\prime}(r) \geqq 0$ and

$$
\alpha(r)= \begin{cases}0 & (r \leqq 1) \\ 1 & (r \geqq 2)\end{cases}
$$

Then we have

$$
\begin{aligned}
\frac{\varphi}{r}-\frac{\partial \varphi}{\partial r} & =\frac{1+r}{r}(1+r)^{-1+\varepsilon}-\varepsilon(1+r)^{-1+\varepsilon} \\
& \geqq(1-\varepsilon)(1+r)^{-1+\varepsilon}>0 \quad(r \geqq 2),
\end{aligned}
$$

and

$$
\begin{aligned}
\frac{1}{2} \frac{\partial \varphi}{\partial r} & =\frac{\varepsilon}{2} \alpha(r)(1+r)^{-1+\varepsilon}+\frac{1}{2} \alpha^{\prime}(r)(1+r)^{\varepsilon} \\
& \geqq \frac{\varepsilon}{2} \alpha(r)(1+r)^{-1+\varepsilon} \geqq 0 \quad(r>0) .
\end{aligned}
$$

With the $\varphi$ defined above we can apply Lemma 5.2.1. The left hand side of (5.2.1) is estimated from below by

$$
\frac{\varepsilon}{2} \int_{B_{R}} \alpha(r)(1+r)^{-1+\varepsilon}|\mathscr{D} v|^{2} d x-2 \sup _{1 \leqq r \leqq 2}\left|\frac{\varphi}{r}-\frac{\partial \varphi}{\partial r}\right| \int_{B_{12}}|\mathscr{D} v|^{2} d x .
$$

Let $\varepsilon$ be sufficiently small. The sum of the volume integrals of the right hand side of (5.2.1) is estimated by 
$(5.2 .5)$

$$
\begin{aligned}
& C\left\{\int_{B_{R}}(1+r)^{-1-\delta+\varepsilon}|v|^{2} d x\right. \\
& \quad+\kappa_{2}\left[\int_{B_{R}}(1+r)^{2 \delta-(1+\varepsilon)}|v|^{2} d x\right]^{1 / 2}\left[\int_{B_{R}}(1+r)^{1-4 \delta+38}|v|^{2} d x\right]^{1 / 2} \\
& \quad+\left[\int_{B_{R}} \alpha(r)(1+r)^{-1+\varepsilon}|\mathscr{D} v|^{2} d x\right]^{1 / 2} \\
& \quad+\left|\int_{B_{R}} \varphi f_{1} \overline{\mathscr{D}_{r} v} d x\right| \\
& \leq C \\
& \quad+\left[\int_{B_{R}} \alpha(r)\left(1+\left.r\right|_{-(1+\varepsilon) / 2} ^{2}+\kappa_{2}|| v\left\|_{\delta-(1+\varepsilon) / 2}|| v\right\|_{-(1+\varepsilon) / 2}(1+r)^{-1+\varepsilon}|\mathscr{D} v|^{2} d x\right]^{1 / 2}\left(\|v\|_{-(1+\varepsilon) / 2}+1\right)\right\} \\
& \left.\left.\left.\quad+\mid \int_{B_{R}} \varphi f_{1} \overline{\mathscr{D}_{r} v} d x\right]^{1 / 2}+\left[\int_{B_{R}}(1+r)^{1+\varepsilon}\left|f_{2}\right|^{2} d x\right]^{1 / 2}\right)\right\} \\
& \leqq \eta \int_{R^{n}} \alpha(r)(1+r)^{-1+\varepsilon}|\mathscr{D} v|^{2} d x+C_{\eta}\left\{\|v\|_{-(1+\varepsilon) / 2}^{2}+1\right\} \\
& \quad+\left|\int_{B_{R}} \varphi f_{1} \overline{\mathscr{D}_{r} v} d x\right|,
\end{aligned}
$$

for any $\eta>0$, where we used Corollory 5.1.3, the fact that $f_{2} \in L_{2,(1+\varepsilon) / 2}$ and $\delta>\frac{1}{2}$. We can apply Lemma 5.2.2 to estimate the last term of (5.2.5) to obtain

$$
\begin{aligned}
& \left|\int_{B_{R}} \varphi f_{1} \overline{\mathscr{D}_{r} v} d x\right| \\
& \leqq\left|\int_{S_{R}} \varphi f_{1} \bar{v} d S\right| \\
& +C\left\{\left\|\left(\frac{\partial}{\partial r}+\frac{n-1}{2 r}-i k\right) \phi_{1}\right\|-(1+\varepsilon) / 2\left(\|v\|_{(-1-2 \delta+3 \varepsilon) / 2}+\|v\|_{(1-4 \delta+3 \varepsilon) / 2}\right)\right. \\
& +\left\|\phi_{1}\right\|_{-(1+\varepsilon) / 2}\|v\|_{(-3-2 \delta+3 \varepsilon) / 2} \\
& \left.+\kappa_{2}\|v\|_{\delta-(1+\varepsilon) / 2}\left\|f_{1}\right\|_{-\delta+1 / 2+3 \varepsilon / 2}\right\} \\
& \leqq \int_{S_{R}} \varphi\left|f_{1}\right||v| d S+C\|v\|_{-(1+\varepsilon) / 2} \\
& +C\left\{\|\mathscr{D} v\|_{(-1+\varepsilon) / 2, E_{2}}+\|v\|_{-(1+\varepsilon) / 2}+1\right\}\left\|f_{1}\right\|_{\delta-(1+\varepsilon) / 2} \\
& \leqq \int_{S_{R}} \varphi\left|f_{1}\right||v| d S+\eta \int \alpha(r)(1+r)^{-1+\varepsilon}|\mathscr{D} v|^{2} d x \\
& +C_{\eta}\left\{\|v\|_{-(1+\varepsilon) / 2}^{2}+1\right\}
\end{aligned}
$$

for any $\eta>0$, where we used again Corollary 5.1.3. 
Combining these three estimates, we have

$$
\begin{aligned}
& \frac{\varepsilon}{2} \int_{B_{R}} \alpha(r)(1+r)^{-1+\varepsilon}|\mathscr{D} v|^{2} d x-2 \eta \int \alpha(r)(1+r)^{-1+\varepsilon}|\mathscr{D} v|^{2} d x \\
& \quad \leqq C_{\eta}\left\{I_{S}(R)+\int_{B_{12}}|\mathscr{D} v|^{2} d x+\|v\|_{-(1+\varepsilon) / 2}^{2}+1\right\} \\
& \quad \leqq C_{\eta}\left\{I_{S}(R)+\|v\|_{-(1+\varepsilon) / 2}^{2}+1\right\},
\end{aligned}
$$

where we put

$$
I_{S}(R)=\int_{S_{R}}(1+r)^{2}\left\{|\mathscr{D} v|^{2}+\left|f_{1}\right|^{2}+|v|^{2}\right\} d S
$$

and used the estimate

$$
\begin{aligned}
\int_{B_{12}}|\mathscr{D} v|^{2} d x & \leqq C\left\{\|v\|_{B_{3}}^{2}+\|f\|_{B_{3}}^{2}\right\} \\
& \leqq C\left\{\|v\|_{-(1+\varepsilon) / 2}^{2}+1\right\},
\end{aligned}
$$

wich follows from Lemma 2.1 of [9].

Since $\mathscr{D} v, f_{1}, v \in L_{2, \varepsilon / 2}\left(E_{1}\right)$, Lemma 5.1.1 shows that

$$
\lim _{R \rightarrow \infty} I_{S}(R)=0 \text {. }
$$

Take the inferior limit of the both sides of (5.2.7) and let $\eta$ be sufficiently small. Then we have (4.3.3).

5.3. Proof of Lemma 4.3.2. The definition of $\mathscr{D}_{r}$ enables one to write

$$
\left|\mathscr{D}_{r} v\right|^{2}=\left|\frac{\partial v}{\partial r}+\frac{n-1}{2 r}+\kappa_{2} v\right|^{2}+\kappa_{1}^{2}|v|^{2}+2 \kappa_{1} \operatorname{Im}\left[\frac{\partial v}{\partial r} \vec{v}\right]
$$

which, integrated over the sphere $S_{R}$ gives

$$
\kappa_{1}^{2} \int_{S_{R}}|v|^{2} d S \leqq \int_{S_{R}}\left|\mathscr{D}_{r} v\right|^{2} d S-2 \kappa_{1} \operatorname{Im} \int \frac{\partial v}{\partial r} \bar{v} d S
$$

Employing the equation (5.1.7) with $\varphi \equiv 1$ in the last term of (5.3.1), we have

$$
\begin{aligned}
\kappa_{1}^{2} \int_{S_{R}}|v|^{2} d S & \leqq \int_{S_{R}}\left|\mathscr{D}_{r} v\right|^{2} d S-4 \kappa_{1}^{2} \kappa_{2} \int_{B_{R}}|v|^{2} d x+2 \kappa_{1} \operatorname{Im} \int_{B_{R}} f \bar{v} d x \\
& \leqq \int_{S_{R}}\left|\mathscr{D}_{r} v\right|^{2} d S+2 \kappa_{1} \operatorname{Im} \int_{B_{R}} f \bar{v} d x
\end{aligned}
$$

Now remember the definition (4.2.10) of $f$ to obtain

$$
\int_{B_{R}} f \bar{v} d x=\int_{B_{R}}-\left(L-k^{2}\right)\left(U(k) \phi_{1}\right) \bar{v} d x
$$




$$
\begin{aligned}
= & \int_{S_{R}} \frac{\partial}{\partial r}\left(U(k) \phi_{1}\right) \bar{v} d S-\int_{S_{R}} U \phi_{1} \frac{\partial v}{\partial r} d S-\int_{B_{R}} U \phi_{1}\left(\overline{\left.L-k^{2}\right) v} d x\right. \\
= & \text { the above two surface integrals }- \\
& -\int_{B_{R}} U \phi_{1} \bar{f} d x-2 i \kappa_{1} \kappa_{2} \int_{B_{R}} \phi_{1}^{2} d x,
\end{aligned}
$$

where we used the relations (4.2.6) and $\mu=2 i \kappa_{1} \kappa_{2}$. Now,

$$
\begin{aligned}
& \operatorname{Im} \int_{B_{R}} U \phi_{1} \bar{f} d x=\operatorname{Im} 2 i \int_{B_{R}} \frac{\partial X}{\partial r} \phi_{1}\left(\frac{\partial}{\partial r}+\frac{n-1}{2 r}+i k\right) \phi_{1} d x \\
= & 2 \int_{B_{R}} \frac{\partial X}{\partial r} \phi_{1}\left(\frac{\partial}{\partial r}+\frac{n-1}{2 r}\right) \phi_{1} d x \\
= & 2 \int_{S_{R}} \frac{\partial X}{\partial r} \phi_{1}^{2} d S-2 \int_{S_{R}} \frac{\partial^{2} X}{\partial r^{2}} \phi_{1}^{2} d S-2 \int_{B_{R}} \frac{\partial X}{\partial r}\left(\frac{\partial}{\partial r}+\frac{n-1}{2 r}\right) \phi_{1} \bullet \phi_{1} d x \\
= & \int_{S_{R}} \frac{\partial X}{\partial r} \phi_{1}^{2} d S-\int_{S_{R}} \frac{\partial^{2} X}{\partial r^{2}} \phi_{1}^{2} d S .
\end{aligned}
$$

Put these two equations into (5.3.2) to obtain

$$
\begin{aligned}
& \kappa_{1}^{2} \int_{S_{R}}|v|^{2} d S \leqq \int_{S_{R}}\left|\mathscr{D}_{r} v\right|^{2} d S+ \\
& \quad+\int_{S_{R}}\left[\left|\frac{\partial}{\partial r}\left(U \phi_{1}\right)\right| \cdot|v|+\left|\phi_{1}\right| \cdot\left|\mathscr{D}_{r} v\right|\right] d S \\
& \quad+C \int_{S_{R}}\left[\left|\phi_{1}\right| \cdot|v|+\phi_{1}^{2}\right] d S
\end{aligned}
$$

which implies

$$
\int_{S_{R}}|v|^{2} d S \leqq C \int_{S_{R}}\left|\mathscr{D}_{r} v\right|^{2} d S+C \int_{S_{R}}\left(\left|\frac{\partial}{\partial r} \phi_{1}\right|^{2}+\left|\phi_{1}\right|^{2}\right) d S
$$

Multiply both sides of this equation by $(1+R)^{-1-\varepsilon}$ and integrate from $\rho$ to $\infty$ with respect to $R$ to obtain (4.3.4) with $\alpha(\rho)=C\left[\left\|\frac{\partial}{\partial r} \phi_{1}\right\|-(1+\varepsilon) / 2, E_{\mathbf{p}}+\left\|\phi_{1}\right\|_{-(1+\varepsilon) / 2, E_{\mathbf{p}}}\right]$.

Q.E.D.

\section{References}

[1] Agmon, S., Spectral properties of Schrödinger operators and scattering theory. Ann. Scuola Nor. Sup. Pisa (4)2 (1975), 151-218.

[2] Gel'fand, I. M. and Silov, G. E., Generalized functions, 3, Moskow, 1958; English transl. Academic Press, New York-London, 1967.

[3] Gel'fand, I. M. and Vilenkin, N. Ya., Generalized functions, 4, Moskow, 1961; English transl. Academic Press, New York-London, 1964.

[4] Hörmander, L., The existence of wave operators in scattering theory, Math. Z. 146 (1976), 69-91. 
[5] Ikebe, T., Eigenfunction expansions associated with the Schrödinger operators and their applications to scattering theory, Arch. Rat. Mech. Anal. 5 (1960), 1-34.

[6] - Wave operators and spectral representations, Proceedings of a conference held at Katata (1972), 150-164. (Japanese).

[7] — Spectral representation for Schrödinger operators with long-range potentials. J. Functional Analysis 20 (1975), 158-177.

[8] - Spectral representation for Scrödinger operators with long-range potentials, II-Perturbation by short-range potentials-. Publ. RIMS Kyoto Univ., 且1 (1976), 551558.

[9] Ikebe, T. and Y. Saitō, Limiting absorption method and absolute continuity for the Schrödinger operator. J. Math. Kyoto Univ. 7 (1972), 513-542.

[10] Isozaki, H., On the long-range stationary wave operators, PRIMS Kyoto Univ., 13 (1977), 589-626.

[11] Kato, T., Perturbation theory for linear operators, Springer, Berlin-New York, 1966.

[12] Kato, T. and S. T. Kuroda, The abstract theory of scattering, Rocky Mountain J. Math. 1 (1971), 127-171.

[13] Kuroda, S. T., Construction of eigenfunction expansions by the perturbation method and its application to n-dimensional Schrödinger operators, Technical Report 744 , Mathematics Research Center, Univ. of Wisconsin, Madison, Wis., 1967.

[14] Pinchuk, G., Abstract time-independent wave operator theory, for long-range potentials, to appear.

[15] Saitō, Y., Spectral theory for second-order differential operators with long-range operator valued coefficients I, Japan J. Math. 1 (1975), 311-349; II, ibid 351-382.

[16] — Eigenfunction expansions for Schrödinger operators with long-range potentials $Q(y)=O\left(|y|^{-8}\right) \varepsilon>0$, Osaka J. Math. 14 (1977), 37-53.

[17] Thoe, D. W., Eigenfunction expansions associated with Schrödinger operators in $R_{n}$, $n \geqq 4$, Arch. Rat. Mech. Anal. 26 (1967), 335-356. 
